\title{
Using Curvature Power To Map the Domain of Inverse Micellar Cubic Phases: The Case of Aliphatic Aldehydes in 1,2-Dioleoyl-sn-glycero-3- phosphoethanolamine
}

\author{
Jamie Burrell, $^{\dagger}$ Marcus K. Dymond, ${ }^{*}+\infty$ Richard J. Gillams, $^{\dagger}$ Duncan J. Parker, ${ }^{\dagger}$ G. John Langley, ${ }^{\dagger}$ \\ Ana Labrador, ${ }^{\S}$ Tommy Nylander, ${ }^{\| \odot}$ and George S. Attard ${ }^{*} \dagger$ \\ ${ }^{\dagger}$ Chemistry, Faculty of Natural and Environmental Sciences, University of Southampton, Southampton SO17 1BJ, United Kingdom \\ ${ }^{\ddagger}$ Division of Chemistry, School of Pharmacy and Biomolecular Sciences, University of Brighton, Brighton, BN2 4GJ, United Kingdom \\ ${ }^{\S}$ MAX IV Laboratory, Lund University, P.O. Box 118, SE-221 00, Lund, Sweden \\ "Physical Chemistry, Lund University, P.O. Box 124, SE-221 00, Lund, Sweden
}

\section{Supporting Information}

\begin{abstract}
Oxylipins, or fatty aldehydes, are a class of molecules produced from membrane lipids as a result of oxidative stress or enzyme-mediated peroxidation. Here we report the effects of two biologically important fatty aldehydes, trans,trans-2,4-decanedienal (DD) and cis-11-hexadecenal (HD), on the phase behavior of the lipid 1,2-dioleoyl-sn-glycero-3-phosphoethanolamine (DOPE) in water. We compare the phase behavior of $\mathrm{DD} / \mathrm{DOPE}$ and $\mathrm{HD} /$ DOPE mixtures to the phase behavior of oleic acid/DOPE mixtures and show that $\mathrm{DD}, \mathrm{HD}$, and oleic acid have similar effects on the phase diagrams of DOPE. Notably, both DD and HD, like oleic acid, induce the formation of $F d 3 m$ inverse micellar cubic phases in DOPE/water mixtures. This is the first time that $F d 3 m$ phases in

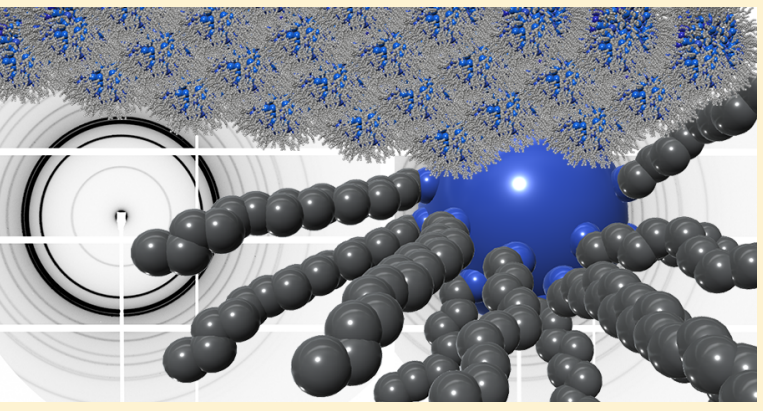
fatty aldehyde-containing mixtures have been reported. We assess the effects of DD, HD, and oleic acid on DOPE in terms of lipid spontaneous curvatures and propose a method to predict the formation of $F d 3 m$ phases from the curvature power of amphiphiles. This methodology predicts that $F d 3 m$ phases will become stable if the spontaneous curvature of a lipid mixture is $-0.48 \pm 0.05 \mathrm{~nm}^{-1}$ or less.
\end{abstract}

\section{INTRODUCTION}

Medium- to long-chain aliphatic aldehydes (fatty aldehydes) are a complex and biologically important set of compounds, termed oxylipins, that are produced from unsaturated membrane lipids in a wide range of organisms as a result of enzyme-mediated peroxidation or oxidative stress. ${ }^{1,2}$ Fatty aldehydes and alcohols are intermediates in the synthesis of the major membrane lipid species, and hence do not generally accumulate as free lipids in vivo. Although oxylipins occur at low concentrations there is an increasing body of evidence supporting the view that they play key roles in diverse intracellular, as well as extracellular, communication processes. $^{3}$ For example, in bacterial bioluminescence fatty aldehydes such as tetradecanal, are the preferred substrates for the oxidation of reduced riboflavine phosphate by luciferase. ${ }^{4}$ In plants, aldehydes such as trans-2hexenal, produced as downstream products following the lipoxygenase (LOX)-mediated peroxidation of polyunsaturated fatty acids (PUFAs), appear to be part of a defense system against bacterial attack. $^{5}$ It has also been suggested that oxylipins in fungi influence biochemical processes in infected tissues by mimicking the host's own oxylipins. ${ }^{6}$ Marine diatoms release fatty aldehydes such as $2 E, 4 E$-decanedienal when under environmental stress, either through nutrient limitation (silicon or phosphorus) $)^{7,8}$ or through predation. ${ }^{9}$ There is some evidence that fatty aldehydes released by diatoms during episodes of predation are able to impair the reproduction of predator species. ${ }^{10}$ In humans, poor regulation of intracellular fatty aldehydes either through mutations in the long-chain fatty aldehyde dehydrogenase (ALDH3A2) gene, or inhibition of the enzyme, leads to Sjögren-Larsson Syndrome ${ }^{11}$ or other severe immunological disorders, ${ }^{12}$ with concomitant accumulation of trans-2-hexadecenal.

Because of their hydrophobic character, fatty aldehydes tend to be localized in bilayer membranes. This raises the question of what effects such compounds might have on the physical properties of lipid bilayers, and in particular on their stored curvature elastic energy. Curvature elastic energy has been shown to play a central role in modulating the activity of membrane proteins. It is known that both fatty acids (FA) and fatty alcohols promote the formation of aggregate structures

Received: August 23, 2017

Revised: October 4, 2017

Published: October 5, 2017 
Table 1. Overview of Systems That Form Fd3m Phases Together with Lattice Parameters Determined at the Indicated Compositions and Temperatures ${ }^{a}$

\begin{tabular}{|c|c|c|c|}
\hline components & composition/temperature & lattice parameter $/ \mathrm{nm}$ & ref \\
\hline egg DAG/egg PC & 78.5 wt $\%$ DAG; tris- $\mathrm{HCl} ; 25{ }^{\circ} \mathrm{C}$ & 11.44 & 15 \\
\hline lipid extract $P$. fluorescens & 11.9 wt $\%$ FA, 47.7 wt $\%$ PE, 13.1 wt $\%$ HC & $\sim 13.3$ & 16 \\
\hline 1,3-DOG/DOPC & 0.70 1,3-DOG; xs. $\mathrm{H}_{2} \mathrm{O} ; 30^{\circ} \mathrm{C}$ & $15.3 \pm 0.2$ & 17 \\
\hline 1,2-DOG/DOPC & 0.77 1,2-DOG; xs. $\mathrm{H}_{2} \mathrm{O} ; 30^{\circ} \mathrm{C}$ & 13.1 & 17 \\
\hline 1,3-DOG/eggPC & 0.67 1,3-DOG; xs. $\mathrm{H}_{2} \mathrm{O} ; 25^{\circ} \mathrm{C}$ & 15.9 & 17 \\
\hline $\mathrm{NaOA} / \mathrm{OA}$ & $0.21-0.41 \mathrm{NaOA} ;$ xs. $\mathrm{H}_{2} \mathrm{O}$ & $15.3 \pm 0.2$ & 18 \\
\hline $\mathrm{OA} / \mathrm{MO}$ & $0.71 \mathrm{OA} ; 20{ }^{\circ} \mathrm{C}$ & 13.98 & 19 \\
\hline $\mathrm{OA} / \mathrm{MO}$ & $0.60 \mathrm{OA} ; 40^{\circ} \mathrm{C}$ & 16.69 & 19 \\
\hline MA/DMPC & $54^{\circ} \mathrm{C}$ & 17.01 & 19 \\
\hline DOG/DOPE & $42^{\circ} \mathrm{C}$ & 13.43 & 19 \\
\hline DPG/DPPC & $1: 2(\mathrm{~mol} / \mathrm{mol}) ; 82{ }^{\circ} \mathrm{C}$ & $16.3 \pm 0.1$ & 20 \\
\hline $\mathrm{C}_{14} \mathrm{OH} / \mathrm{PC}$ & xs. $\mathrm{H}_{2} \mathrm{O} ; 0.80 \mathrm{C}_{14} \mathrm{OH} ; 90{ }^{\circ} \mathrm{C}$ & 17.8 & 21 \\
\hline $\mathrm{C}_{16} \mathrm{OH} / \mathrm{PC}$ & xs. $\mathrm{H}_{2} \mathrm{O} ; 0.80 \mathrm{C}_{16} \mathrm{OH} ; 90{ }^{\circ} \mathrm{C}$ & 17.7 & 21 \\
\hline $\mathrm{C}_{18} \mathrm{OH} / \mathrm{PC}$ & xs. $\mathrm{H}_{2} \mathrm{O} ; 0.86 \mathrm{C}_{18} \mathrm{OH} ; 90{ }^{\circ} \mathrm{C}$ & 18.4 & 21 \\
\hline $\mathrm{C}_{20} \mathrm{OH} / \mathrm{PC}$ & xs. $\mathrm{H}_{2} \mathrm{O} ; 0.78 ; \mathrm{C}_{20} \mathrm{OH} ; 90{ }^{\circ} \mathrm{C}$ & 19.9 & 21 \\
\hline DHOXPG & xs. $\mathrm{H}_{2} \mathrm{O} ; 120{ }^{\circ} \mathrm{C}$ & $14.8 \pm 0.1$ & 22 \\
\hline MA/DMPC & $85 \% \mathrm{MA} ; 70{ }^{\circ} \mathrm{C}$ & 14.5 & 23 \\
\hline LA/DLPC & $85 \% \mathrm{LA} ; 40{ }^{\circ} \mathrm{C}$ & 15.0 & 23 \\
\hline DPhOGG & xs. $\mathrm{H}_{2} \mathrm{O} ;-20{ }^{\circ} \mathrm{C}-80{ }^{\circ} \mathrm{C}$ & 14.8 & 24 \\
\hline DOG/DOPE & 0.60 DOG; > 25 wt $\% \mathrm{H}_{2} \mathrm{O} ; 25^{\circ} \mathrm{C}$ & 15.0 & 25 \\
\hline limonene/MO & 0.4 limonene; $\geq 12.5 \mathrm{H}_{2} \mathrm{O} ; 23{ }^{\circ} \mathrm{C}$ & 16.5 & 26 \\
\hline vitE/soyPC & 0.65 vitE & 14.8 & 27 \\
\hline vitE/soyPC/polysorbate 80 & 90 wt $\% \mathrm{H}_{2} \mathrm{O}$ & 19.0 & 27 \\
\hline bovine liver PI/DOPC & $0.1 \mathrm{PI} ; 30$ wt $\% \mathrm{H}_{2} \mathrm{O} ; 37^{\circ} \mathrm{C}$ & $16.1 \pm 0.24$ & 28 \\
\hline wheat germ PI/DOPC & $0.11 \mathrm{PI} ; 30$ wt $\% \mathrm{H}_{2} \mathrm{O} ; 37^{\circ} \mathrm{C}$ & $16.7 \pm 0.39$ & 28 \\
\hline vitE/MO/Asc & 35 wt $\%$ vitE; 4 wt $\%$ Asc; $25{ }^{\circ} \mathrm{C}$ & 8.19 & 29 \\
\hline $\mathrm{EA} / \mathrm{ME}$ & 50 wt \% EA; PBS; $26{ }^{\circ} \mathrm{C}$ & 15.23 & 30 \\
\hline $\mathrm{OA} / \mathrm{ME}$ & 60 wt \% OA; PBS; $26^{\circ} \mathrm{C}$ & 14.63 & 30 \\
\hline $\mathrm{OA} / \mathrm{DOPC}$ & $70-80$ wt $\%$; water $25-60{ }^{\circ} \mathrm{C}$ & $14-18$ & 31 \\
\hline OA/DOPE & $50-70$ wt $\%$ water $25-60{ }^{\circ} \mathrm{C}$ & $14-18$ & \\
\hline
\end{tabular}

${ }^{a}$ DAG, diacylgycerol; PC, phosphatidylcholine; PE, phosphatidylethanolamine; HC, hydrocarbon; 1,2-DOG, 1,2-dioleoyl-sn-glycerol; 1,3-DOG, 1,3dioleoyl-sn-glycerol; DOPC, 1,2-dioleoyl-sn-glycero-3-phosphocholine; NaOA, sodium oleate; OA, oleic acid; MO, monoolein; MA, myristic acid; DMPC, 1,2-dimyristoyl-sn-glycero-3-phosphocholine; DOPE, 1,2-dioleoyl-sn-glycero-3-phosphoethanolamine; DOG, dioleoylglycerol; 1,2-DPG, 1,2dipalmitoyl-sn-glycerol; DPPC, 1,2-dipalmitoyl-sn-glycero-3-phosphocholine; $\mathrm{C}_{14} \mathrm{OH}$, $n$-tetradecanol; $\mathrm{C}_{18} \mathrm{OH}, n$-octadecanol; $\mathrm{C}_{20} \mathrm{OH}$, $n$-eicosanol; DHOXPG, 1,2-di-O-hexadecyl-3-O-( $\alpha$ or $\beta$ )-D-xylopyranosyl)-sn-glycerol; LA, lauric acid; DLPC, 1,2-dilauroyl-sn-glycero-3-phosphocholine; DPhOGG, 1,3-di-O-phytanyl-2-O-( $\beta$-gycosyl)-sn-gycerol; vitE, $\alpha$-tocopherol; soyPC, soy phosphatidylcholine; PI, phosphatidylinositol; Asc, ascorbic acid; EA, elaidic acid; ME, monoelaidin.

with large negative mean curvatures, when added to phosphatidylcholine (PC) lipids, which represent the most abundant lipid class in eukaryotic cells. It is also well established that FA can increase the activity of membrane-associated proteins when added to PC vesicles, often by increasing curvature elastic energy. ${ }^{13,14}$ The promotion of a large negative curvature by FA is evidenced by the formation of $\mathrm{Fd} 3 \mathrm{~m}$ inverse micellar cubic phases, stable in excess water, in PC/FA binary mixtures.

The $F d 3 m$ inverse micellar phase, first observed in lipid extracts of Pseudomonas fluorescens, has now been shown to occur in a range of binary lipid mixtures, as well as in single component glycolipids, as summarized in Table 1 .

The spontaneous curvature $\left(c_{0}\right)$ of a lipid, i.e., the inverse of the spontaneous radius of curvature $\left(R_{0}\right)$ of a lipid aggregate in the unstressed state, ${ }^{32,33}$ is an important parameter that provides a means to quantify membrane stored elastic energy. ${ }^{18,34} c_{0}$ thus enables quantitative models of lipidprotein, lipid-DNA interactions and phospholipid homeostasis to be assembled. ${ }^{14,35-37}$ Lipid spontaneous curvatures are determined from the structural parameters of the inverse hexagonal $\left(\mathrm{H}_{\mathrm{II}}\right)$ lyotropic liquid crystal phase of lipids using small-angle X-ray diffraction (SAXRD).$^{38-43}$ By definition $R_{0}$ is the measured distance from the lipid headgroup and water interface to the neutral plane, ${ }^{44}$ where the bending and stretching modes of the lipid monolayer are decoupled. In practice, it is commonplace to measure $R_{0}$ at the pivotal plane, where the area per lipid does not change with phase curvature and is therefore easier to determine experimentally. However, it is worthwhile pointing out that where good quality electron density data are available, the neutral plane position can be located relative to the pivotal plane. ${ }^{45}$ We recently reported a method for estimating the spontaneous curvature of lipids at the pivotal plane from temperature-induced changes in the lattice parameters of $\mathrm{H}_{\mathrm{II}}$ phases that are formed when they are mixed at low mole fractions in DOPE. ${ }^{46}$ With this new method, see Supporting Information for a summary, we determined the spontaneous curvature at the pivotal plane of trans,trans-2,4decanedienal (DD) and cis-11-hexadecenal (HD) to be -0.63 \pm 0.05 and $-0.52 \pm 0.04 \mathrm{~nm}^{-1}$, respectively. These values are similar in magnitude to the spontaneous curvature of $\mathrm{OA}-0.71$ $\pm 0.03 \mathrm{~nm}^{-1}$ and since OA forms $\mathrm{Fd} 3 \mathrm{~m}$ phases with DOPE and $\mathrm{DOPC}^{31}$ we suggested that $\mathrm{DD}$ and $\mathrm{HD}$ would also form the $\mathrm{Fd3m}$ phase in DOPE. In the present study, we test this 

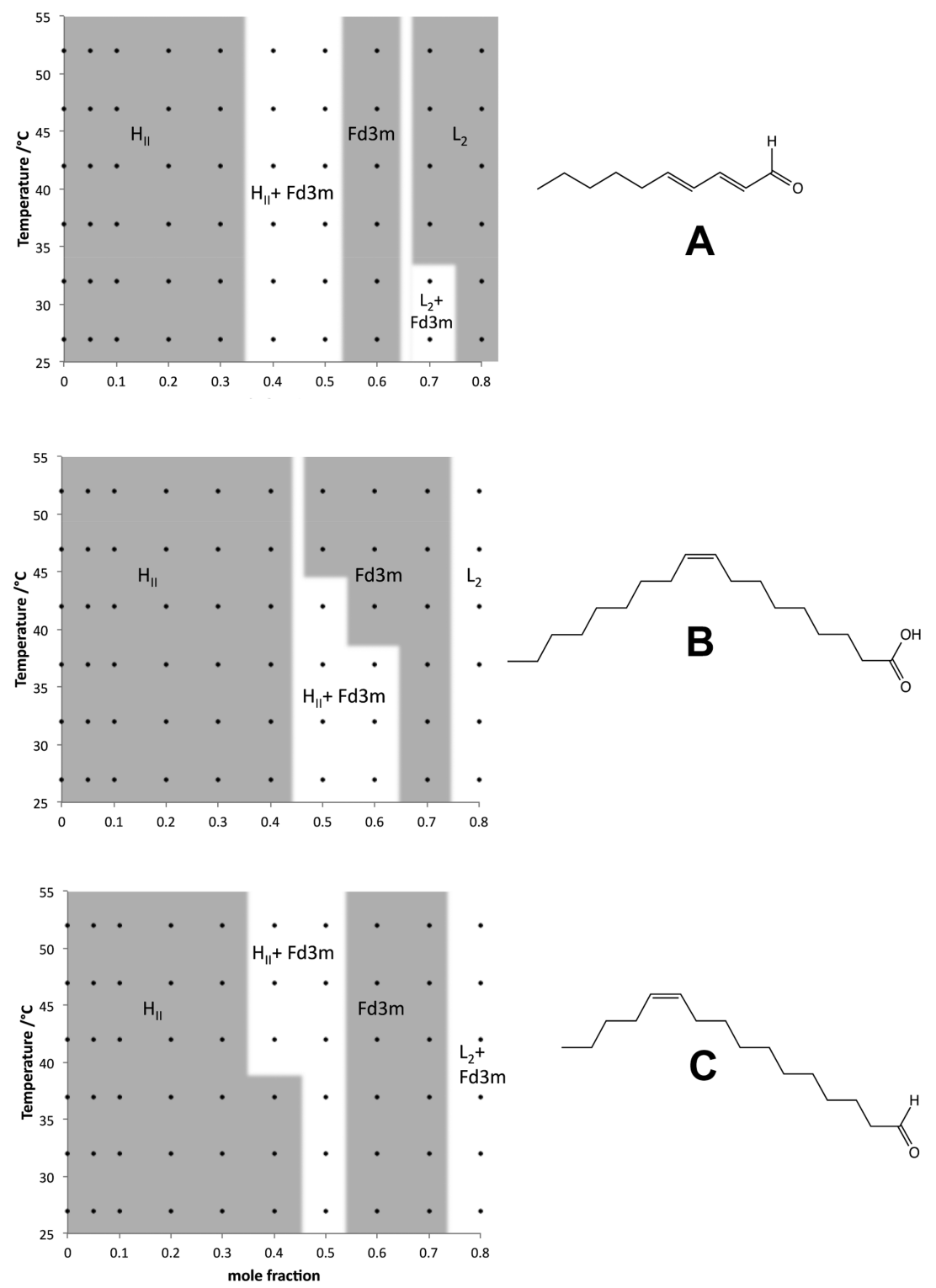

Figure 1. Phase diagrams of binary mixtures of DOPE with trans,trans-2,4-decanedienal (A), oleic acid (B) and cis-11-hexadecenal (C), where the $x$ axis shows the mole fraction of the additive in DOPE. Dots indicate points at which experimental data were acquired.

hypothesis by mapping the temperature/composition phase diagrams of binary mixtures of $\mathrm{DD}$ and $\mathrm{HD}$, with the zwitterionic lipid DOPE. These studies show that both DD and HD do indeed promote the formation of $F d 3 m$ phases. To the best of our knowledge this is the first time that $F d 3 m$ phases in fatty aldehyde-containing mixtures have been reported. We rationalize the phase behavior of the binary mixtures in terms of the estimated spontaneous curvature of DD and HD and compare data to the binary phase diagram of DOPE and OA we have previously published. ${ }^{31}$

\section{EXPERIMENTAL SECTION}

DOPE was purchased from Avanti Polar Lipids (Alabama USA). Chloroform was purchased from Sigma-Aldrich (UK). DD and HD were purchased from Tokyo Chemical Industry U.K. Ltd. Ultrapure water of $18.2 \mathrm{~m} \Omega$ conductivity (Barnstead Nanopure Diamond) was used for the preparation of all samples.

Preparation of Binary Lipid Mixture for Polarizing Optical Microscopy and XRD Measurements. Binary lipid mixtures were prepared in $1.5 \mathrm{~mL}$ microcentrifuge tubes. Dry quantities of DOPE (used as received), typically in the range of 50 to $100 \mathrm{mg}$ were weighed into the tube. The secondary lipid DD or HD was dissolved in chloroform and the appropriate microlitre volumes of $\mathrm{DD}$ or $\mathrm{HD}$ solution with known concentration were added. A further volume of chloroform $(200 \mu \mathrm{L})$ was added to each sample to dissolve all the lipids, which were mixed by vortexing before being dried overnight in vacuo. We estimate the uncertainty in the composition of the lipid mixtures is up to $2 \mathrm{~mol} \%$, based on an estimated DOPE moisture content of $\leq 0.1 \% \mathrm{w} / \mathrm{w}$.

To prepare lyotropic liquid crystal phases at limiting hydration in excess water, $100 \mu \mathrm{L}$ of pure water was added to each dry lipid sample. All samples were mixed manually using a small spatula for several minutes prior to centrifugation at $17000 \mathrm{~g}$ (Heraeus Pico 17 Centrifuge) for $5 \mathrm{~min}$. Manual mixing and centrifugation cycles were repeated three times before samples were incubated at $37{ }^{\circ} \mathrm{C}$ for $2-3$ days. Prior to data collection, a further manual mixing and centrifugation cycle was carried out.

Polarized Optical Microscopy Studies of Binary Lipid Mixtures. The phase behavior of the binary lipid mixtures was initially mapped as a function of temperature and composition by identification of the optical textures of the liquid crystalline phases using polarized light microscopy. Samples prepared as detailed above 

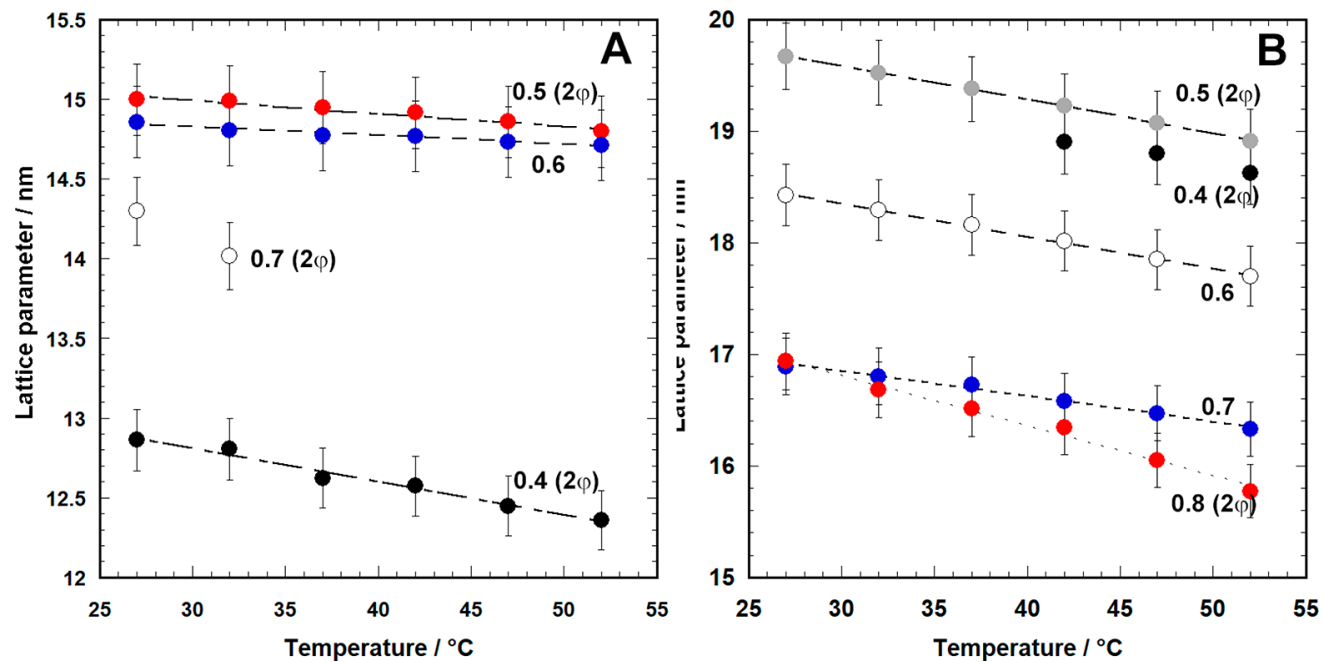

Figure 2. Temperature dependence of the lattice parameters of $F d 3 m$ phases. Panel A shows data for DD/DOPE mixtures and panel B shows data for $\mathrm{HD} / \mathrm{DOPE}$ mixtures at limiting hydration in excess water. " $2 \varphi$ " indicates mixtures with two coexisting phases. Table 2 shows the coefficients of the linear fits used.

were sandwiched between a coverslip and glass slide and observed using an Olympus BH-2 polarizing optical microscope equipped with a Linkham Scientific Instruments THM600 hot stage. The accuracy of the temperature of the hot stage was $\pm 0.2{ }^{\circ} \mathrm{C}$. Phases were identified by their characteristic optical textures. ${ }^{47}$

SAXRD Studies of Binary Lipid Mixtures. SAXRD studies were performed at the SAXRD station 1911-4 of the MAX IV Synchrotron, Lund, Sweden. Extensive details of the experimental setup have been published previously. ${ }^{31,48}$ Figure S1 shows a representative SAXD image and Table $S 1$ provides the lattice parameter data for all the samples we investigated.

Mass Spectrometry Studies. Binary mixtures were investigated by mass spectrometry for the possible formation of Schiff bases through the reaction of the aldehyde and DOPE. Samples were analyzed using a Waters (Manchester, U.K.) Acquity $\mathrm{UPC}^{2}$ TQD tandem quadrupole mass spectrometer and introduced using a $2 \mu \mathrm{L}$ Partial Loop with Needle Overfill (PLNO) injection. Ultrahigh performance supercritical fluid chromatography (UHPSFC) was undertaken using a UPC $^{2}$ Torus Diol column (Waters, $100 \mathrm{~mm} \times$ $3.0 \mathrm{~mm} 1.7 \mu \mathrm{m})$.

Gradient elution from $98 \% \mathrm{CO}_{2} / 2 \%$ methanol modifier $(25 \mathrm{mM}$ ammonium acetate) to $60 \% \mathrm{CO}_{2} / 40 \%$ methanol modifier $(25 \mathrm{mM}$ ammonium acetate) was performed over $10 \mathrm{~min}$ at an eluent flow rate of $1.5 \mathrm{~mL} / \mathrm{min}$ with an Active Back Pressure Regulator (ABPR) pressure of 150 bar. A makeup flow solvent (methanol $1 \%$ formic acid) was pumped at a flow rate of $0.45 \mathrm{~mL} / \mathrm{min}$ into the mass spectrometer. Low-resolution positive ion electrospray ionization mass spectra were recorded.

\section{RESULTS AND DISCUSSION}

Range of Stability of $\mathrm{H}_{\|}$and $F d 3 m$ Phases. The temperature/composition phase diagram of the DD/DOPE binary mixture in excess water as determined by SAXRD is shown in Figure 1A. At low concentrations $(\leq 0.3$ mole fraction) of $\mathrm{DD}$, the system forms an $\mathrm{H}_{\mathrm{II}}$ phase. This phase is stable over the temperature range studied $\left(25\right.$ to $\left.55^{\circ} \mathrm{C}\right)$. At $\mathrm{DD}$ mole fractions of 0.4 and 0.5 , the system becomes biphasic with the $\mathrm{H}_{\text {II }}$ phase coexisting with an optically isotropic, viscous phase that is identified as an $F d 3 m$ inverse micellar cubic phase from SAXRD. The mixture with a DD mole fraction of 0.6 forms a homogeneous $F d 3 m$ phase. At higher concentrations and at the lower temperatures, a new biphasic region forms, consisting of the $F d 3 m$ phase in equilibrium with the highly fluid inverse micellar phase $\left(\mathrm{L}_{2}\right)$, which gives way to a single $\mathrm{L}_{2}$ phase at a DD mole fraction of 0.8 .

The phase behavior of the DD/DOPE system is very similar to that of the OA/DOPE system (Figure 1B), redrawn from Gillams et al., ${ }^{31}$ both in terms of the sequence of phases observed and the ranges of composition over which they occur. In the case of $\mathrm{FA} / \mathrm{PE}$, as well as $\mathrm{FA} / \mathrm{PC}$, mixtures the formation of an $F d 3 m$ phase has been attributed to the existence of a 2:3 phospholipid/FA complex, based on infrared spectroscopy data. ${ }^{23}$ In the DD/DOPE system, the onset of the $F d 3 m$ phase first appears at a mole fraction within the range $0.3<x<0.4$, which is a little lower than for the OA/DOPE system $(0.4<x<$ $0.5)$. The DD system exhibits an $L_{2}$ phase that is stable over a more extensive range of temperatures and compositions compared with the OA system. Taken together these observations suggest that $\mathrm{DD}$ induces a slightly tighter negative interfacial mean curvature than is the case for OA.

The temperature/composition phase diagram of the HD/ DOPE mixture is shown in Figure 1C. The Fd3m phase appears at $\mathrm{HD}$ mole fractions within the range $0.4<x<0.5$ and is present up to the highest concentration of HD studied $(x=$ 0.8 ). The onset of the $F d 3 m$ phase is broadly at compositions that are coincident with those at which it occurs for the OA/ DOPE system. However, in the latter system the Fd3m phase gives way to a single $\mathrm{L}_{2}$ phase when the OA mole fraction is 0.8 . These comparisons suggest that $\mathrm{HD}$ induces a less tight negative interfacial mean curvature than OA.

The similarity between the phase diagrams of the three systems DD/DOPE, OA/DOPE, HD/DOPE indicates that despite the chemical diversity of the two aldehydes studied the same underlying intermolecular interactions underpin the stability of the $F d 3 m$ phase in all three systems. This is an unexpected observation in view of the extensive literature on the formation of Schiff bases between aldehydes and DOPE. ${ }^{49-52}$ Indeed, such adducts have been implicated in the potent biological activity of fatty aldehydes. ${ }^{53,54}$

To determine the extent to which Schiff bases might contribute to the phase behavior we observed, we investigated the mixtures that were used for SAXRD studies by mass spectrometry. While all samples showed the presence of the DOPE parent molecular ion $(m / z: 744)$, we were unable to 
Table 2. Coefficients of Fits of the Lattice Parameters $(a)$ of the Fd3m Phases at Different Mole Fractions $(x)$ of Aldehyde to a Linear Temperature $(T)$ Dependence Given by $a=g T+m^{a}$

DD

\begin{tabular}{ccccc}
$\boldsymbol{x}$ & $\boldsymbol{g} / \mathrm{nm} \mathrm{K}^{-1}$ & $\mathrm{~m} / \mathrm{nm}$ & $g / \mathrm{nm} \mathrm{K}^{-1}$ & $\mathrm{~m} / \mathrm{nm}$ \\
\hline 0.4 & $-0.021( \pm 0.001)$ & $19.11( \pm 0.48)$ & $-0.028( \pm 0.001)$ & $27.77( \pm 0.65)$ \\
0.5 & $-0.008( \pm 0.0004)$ & $17.46( \pm 0.43)$ & $-0.030( \pm 0.002)$ & $28.78( \pm 0.69)$ \\
0.6 & $-0.005( \pm 0.0003)$ & $16.47( \pm 0.40)$ & $-0.029( \pm 0.001)$ & $27.20( \pm 0.67)$ \\
0.7 & $\underline{-0.056( \pm 0.003)}$ & $\underline{31.16( \pm 0.50)}$ & $-0.023( \pm 0.001)$ & $23.68( \pm 0.59)$ \\
0.8 & - & - & $\underline{-0.045( \pm 0.003)}$ & $\underline{30.43( \pm 0.71)}$
\end{tabular}

${ }^{a}$ Grayed-out cells indicate data from coexisting $\mathrm{H}_{\mathrm{II}}$ and $\mathrm{Fd} 3 m$ phases; underlined values indicate coexisting Fd3m and $\mathrm{L}_{2}$ phases.
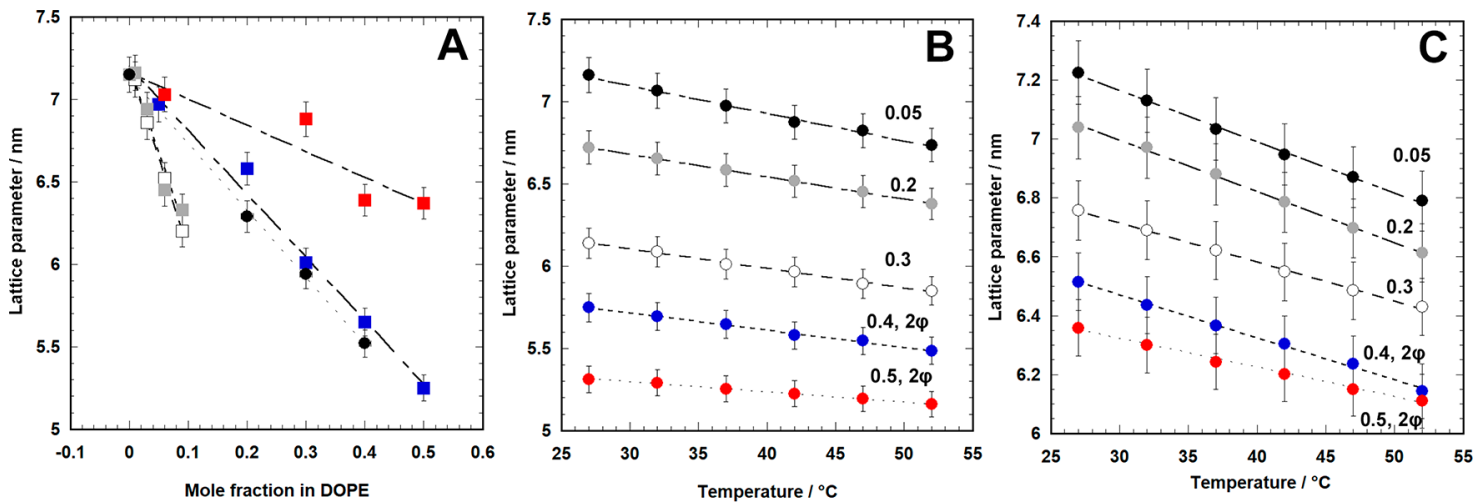

Figure 3. Temperature- and composition-induced changes in the lattice parameters of $H_{\text {II }}$ phases. (A) Effect of mole fraction of DD (blue squares) and $\mathrm{HD}$ (red squares) on the lattice parameter of the $\mathrm{H}_{\mathrm{II}}$ phase of DOPE binary mixtures at $37^{\circ} \mathrm{C}$, all at limiting hydration in water. For comparison $c R$ (white squares), tR (gray squares) and OA (black circles) are also shown. (B,C) Temperature dependence of the lattice parameter of the $\mathrm{H}_{\mathrm{II}}$ phase, at limiting hydration in excess water. Panel B shows DD/DOPE mixtures and Panel C shows HD/DOPE mixtures. Numbers adjacent to points denote mole fraction of DD or HD respectively, " $2 \varphi$ " denotes coexistence of two phases. Table 3 shows the coefficients of the linear fits used.

observe any species with higher $\mathrm{m} / \mathrm{z}$ values such as might be expected for Schiff base products of the type that a previous study reported in mixtures of 1,2 dipalmitoyl-sn-glycero-3phosphoethanolamine and alkanals. ${ }^{49}$ We note that the alkanal to lipid concentration ratio in the Annibal et al. ${ }^{49}$ work was around $4 \times 10^{3}$, whereas in our study this ratio was $\leq 8$. Thus, we can conclude that under the conditions of our experiment Schiff base formation does not occur to any significant extent and so it does not contribute to the phase behavior we observe. This conclusion is consistent with the observation that the phase diagrams of DD/DOPE and HD/DOPE are very similar to that of OA/DOPE in which there is no possibility of Schiff base formation.

Structural Properties of the Fd3m Phase of DD/DOPE and HD/DOPE Mixtures. The dependence of the lattice parameter of the $\mathrm{Fd} 3 \mathrm{~m}$ phase as a function of mixture composition is illustrated in Figure 2 for DD/DOPE and $\mathrm{HD} / \mathrm{DOPE}$ at $52{ }^{\circ} \mathrm{C}$. The lattice parameters of the $\mathrm{Fd} 3 \mathrm{~m}$ phases of the DD/DOPE and HD/DOPE mixtures fall in the range 12.4 to $18.9 \mathrm{~nm}$ over the temperatures and compositions studied. These values are comparable to the lattice parameters previously reported for the $F d 3 m$ phase of OA/DOPE (14-18 $\mathrm{nm}) .^{31}$ Table 1 shows the spread of lattice parameters reported in the literature for $F d 3 m$ phases; these range from 11 to 20 $\mathrm{nm}$. Figure S2 shows the lattice parameter of the $\mathrm{Fd} 3 \mathrm{~m}$ phase as a function of mixture composition at $37^{\circ} \mathrm{C}$, and once again the values we find are consistent with those in Table 1.

Figure 2A,B shows the temperature dependence of the $\mathrm{Fd} 3 \mathrm{~m}$ lattice parameter for DD/DOPE and HD/DOPE mixtures. At all compositions, a linear inverse dependence of lattice parameter on temperature was observed. This relationship is similar to that previously reported for the $F d 3 m$ phase of OA/ DOPE and indicates that HD and DD, like OA, in DOPE mixtures have a negative curvature preference.

Table 2 shows the coefficients of a linear fit to the data in Figure 2A,B. There does not appear to be any trend between the gradient of the temperature-dependent change in lattice parameter with mole fraction of $\mathrm{DD}$ or $\mathrm{HD}$. For both aldehydes, the gradient is typically in the range of -0.5 to $-5 \times 10^{-2} \mathrm{~nm} \mathrm{~K}^{-1}$. It should be noted that many of the data points are for mixtures that have coexisting phases and hence the compositions of the coexisting phases are likely to change as a function of temperature. Single phase systems occur at 0.6 $\mathrm{DD} / \mathrm{DOPE}$ and 0.6 and $0.7 \mathrm{HD} / \mathrm{DOPE}$, and at these compositions gradients of $-0.5 \times 10^{-2},-2.9 \times 10^{-2}$, and $-2.3 \times 10^{-2} \mathrm{~nm} \mathrm{~K}^{-1}$ are observed. The values for the $\mathrm{HD} /$ DOPE $F d 3 m$ phases are consistent with the gradients reported for the temperature-dependent lattice parameter change of the $\mathrm{Fd} 3 \mathrm{~m}$ phases of OA/DOPE mixtures, (circa $-1.5 \times 10^{-2} \mathrm{~nm}$ $\mathrm{K}^{-1}$ at compositions of $0.5,0.6$, and 0.7 wt $\left.\% \mathrm{OA}\right)^{31}$ and fatty alcohol/PC mixtures (circa $-2.3 \times 10^{-2} \mathrm{~nm} \mathrm{~K}^{-1}$ ). ${ }^{21}$ Similarly the lattice parameters of $F d 3 m$ phases in mixtures of limonene and monolinolein vary with temperature with a gradient of roughly $-2.6 \times 10^{-2} \mathrm{~nm} \mathrm{~K}^{-1},{ }^{26}$ while in MA/DMPC mixtures this value is circa $-1.7 \times 10^{-2} \mathrm{~nm} \mathrm{~K}^{-1}$. ${ }^{23}$

Structural Properties of $\mathrm{H}_{\|}$Phases of DD/DOPE and HD/DOPE Mixtures. The OA/DOPE, DD/DOPE, and HD/ DOPE mixtures all exhibit $\mathrm{H}_{\mathrm{II}}$ phases up to an additive mole 
Table 3. Coefficients of Fits of the Lattice Parameters $(a)$ of the $\mathrm{H}_{\mathrm{II}}$ Phases at Different Mole Fractions $(x)$ of Aldehyde to a Linear Temperature $(T)$ Dependence Given by $a=g T+m^{a}$

DD

HD

\begin{tabular}{ccccc}
$\boldsymbol{x}$ & $g / \mathrm{nm} \mathrm{K}^{-1}$ & $\mathrm{~m} / \mathrm{nm}$ & $g / \mathrm{nm} \mathrm{K}^{-1}$ & $\mathrm{~m} / \mathrm{nm}$ \\
\hline 0.05 & $-0.017( \pm 0.008)$ & $7.60( \pm 0.23)$ & $-0.017( \pm 0.002)$ & $7.67( \pm 0.13)$ \\
0.2 & $-0.014( \pm 0.001)$ & $7.10( \pm 0.11)$ & $-0.017( \pm 0.002)$ & $7.51( \pm 0.15)$ \\
0.3 & $-0.012( \pm 0.004)$ & $6.46( \pm 0.30)$ & $-0.014( \pm 0.002)$ & $6.90( \pm 0.13)$ \\
0.4 & $-0.010( \pm 0.004)$ & $6.03( \pm 0.25)$ & $-0.013( \pm 0.003)$ & $6.88( \pm 0.17)$ \\
0.5 & $-0.006( \pm 0.003)$ & $5.48( \pm 0.22)$ & $-0.010( \pm 0.004)$ & $6.61( \pm 0.23)$
\end{tabular}

${ }^{a}$ Grayed out cells indicate compositions at which the $\mathrm{H}_{\text {II }}$ phase coexists with the $\mathrm{F} d 3 \mathrm{~m}$ phase.

fraction of $0.5-0.6$. The lattice parameters of these $\mathrm{H}_{\mathrm{II}}$ phases as a function of additive mole fraction at $37{ }^{\circ} \mathrm{C}$ are shown in Figure $3 \mathrm{~A}$, where we also indicate the values reported in the literature for the $\mathrm{H}_{\mathrm{II}}$ phase of OA/DOPE.

Figure $3 \mathrm{~A}$ shows that the change in lattice parameter of the $\mathrm{H}_{\mathrm{II}}$ phase as a function of increasing DD composition is very close to that for the OA/DOPE system, suggesting that DD and $\mathrm{OA}$ have a similar propensity to induce tighter mean curvature in DOPE $\mathrm{H}_{\mathrm{II}}$ phases. $\mathrm{HD}$ also induces tighter mean curvature in the DOPE $\mathrm{H}_{\mathrm{II}}$ cylinders, shown in Figure $3 \mathrm{~A}$, but to a lesser extent than DD and OA. This is consistent with the previously reported spontaneous curvatures of $\mathrm{DD}, \mathrm{OA}$, and $\mathrm{HD}$, which are $-0.63 \pm 0.05,-0.71 \pm 0.03$, and $-0.52 \pm 0.04$ $\mathrm{nm}^{-1}$, respectively. ${ }^{46}$ However, as the composition of OA, HD, or DD increases and the $\mathrm{H}_{\text {II }}$ phase becomes richer in these molecules, a reduction in the hydrocarbon volume of the $\mathrm{H}_{\mathrm{II}}$ phase might also explain why the shorter DD decreases the $\mathrm{H}_{\mathrm{II}}$ phase lattice parameter more than the longer $\mathrm{HD}$, at an identical mole fraction. This effect would become more significant as the mole fraction of $\mathrm{HD}$ or $\mathrm{DD}$ increases, suggesting that at lower mole fractions the changes are driven by curvature. For comparison, Figure $3 \mathrm{~A}$ shows the composition dependence of the lattice parameter of the $\mathrm{H}_{\mathrm{II}}$ phases for cis-retinal (cR) and trans-retinal (tR) in DOPE. ${ }^{46}$ In both cases the decrease in lattice parameter is steeper as a function of $c R$ or $t R$ concentration than is the case for $\mathrm{OA}, \mathrm{DD}$, or HD. This indicates that that the propensity for the aldehydes $\mathrm{DD}$ and $\mathrm{HD}$ to induce tighter negative curvature is also shared by other hydrophobic aldehydes. The tighter curvature imparted by $\mathrm{cR}$ or $\mathrm{tR}$ compared with $\mathrm{DD}$ or $\mathrm{HD}$ may be due to their wider cross-sectional area, due to the presence of methyl branches. It should be noted that while we cannot completely discount that decreases in the hydrocarbon volume of $\mathrm{cR}$ and $\mathrm{tR}$ may play a role here, measurements were performed with less than $10 \mathrm{~mol} \% \mathrm{cR}$ or $\mathrm{tR}$ in the DOPE host $\mathrm{H}_{\text {II }}$ phase to minimize the changes in lattice parameter due to the reduced hydrophobic volume of the guest. ${ }^{46}$

As expected, and consistent with the behavior of OA/DOPE and $\mathrm{OA} / \mathrm{DOPC}$ mixtures ${ }^{31}$ and $\mathrm{FA} / \mathrm{PC}$ mixtures reported in the literature, ${ }^{23}$ the $\mathrm{H}_{\mathrm{II}}$ phase lattice parameter decreases linearly as a function of temperature for both $\mathrm{DD}$ and $\mathrm{HD}$ (Figure 3B,C) as summarized by the linear fit parameters in Table 3. Figure $S 3$ shows the lattice parameter of the $\mathrm{H}_{\text {II }}$ phase as a function of mixture composition at $52{ }^{\circ} \mathrm{C}$, and once again the values we find are consistent with those in Table 3 .

The lattice parameters of OA/DOPC and OA/DOPE mixtures typically have temperature dependences of $-0.9 \pm$ $0.1 \times 10^{-2}$ and $-1.3 \pm 0.3 \times 10^{-2} \mathrm{~nm} \mathrm{~K}^{-1}$, respectively. ${ }^{31} \mathrm{In}$ general for DD/DOPE mixtures the gradients become slightly less steep as the mole fraction of DD increases, although this trend is less evident in the HD/DOPE mixtures due to data scatter. The values reported in Table 3, which range from -0.6 $\times 10^{-2}$ to $-1.7 \times 10^{-2} \mathrm{~nm} \mathrm{~K}^{-1}$ for DD/DOPE mixtures and from $-1.0 \times 10^{-2}$ to $-1.7 \times 10^{-2} \mathrm{~nm} \mathrm{~K}^{-1}$ for HD/DOPE mixtures are similar to those reported for the $\mathrm{H}_{\text {II }}$ phases of arachidic acid (AA)/1,2-diarachidoyl-sn-glycero-3-phosphocholine (DAPC) $(2: 1)$ (i.e., $\left.-2 \times 10^{-2} \mathrm{~nm} \mathrm{~K}^{-1}\right)$ but smaller than the $-2 \times 10^{-1} \mathrm{~nm} \mathrm{~K}^{-1}$ reported for MA/DMPC (5.7:1). ${ }^{23}$

These observations suggest that like $\mathrm{OA}$, which is probably situated in the hydrocarbon region of the assembly in a protonated form, ${ }^{31}$ the neutral DD and HD are also located in this region. Thus, increased thermal motion of the hydrocarbon chains of DD and HD drives the formation of tighter curvature as temperature increases.

Predicting the Occurrence of $F d 3 m$ Phases from the Curvature Power of Amphiphiles. As indicated in Table 1, the $F d 3 m$ phase occurs in a wide range of lipid systems. We wondered whether there might be a unique metric that might be used to predict whether a given mixture of lipids would form this phase. An obvious measure would be the critical curvature elastic stress that a mixture needs to achieve to form an $F d 3 m$ phase. Assuming, as a zeroth order approximation, that both the mean and Gaussian curvature elastic constants are invariant with respect to molecular structure means that we can seek the critical curvature of a system beyond which $F d 3 m$ phases are expected to form. The mole fractions of guest molecules $\left(x_{j ; \min }\right)$ at which the $F d 3 m$ phases are first observed in a given mixture, together with the respective values of the spontaneous curvatures $\left(c_{0 \text {;host }}, c_{0 \text {; guest }}\right)$, can be used to estimate the magnitude of the spontaneous curvature of the mixture $\left(c_{0 ; \text { crit }}\right)$ at which the $F d 3 m$ phase becomes stable by using eq 1

$$
c_{0 ; \text { crit }}=x_{j ; \text { min }} c_{0 ; \text { guest }}+\left(1-x_{j ; \text { min }}\right) c_{0 ; \text { host }}
$$

Using the data for the OA/DOPC, OA/DOPE, DD/DOPE, and $\mathrm{HD} / \mathrm{DOPE}$ systems gives a value of $c_{0 \text {; crit }}$ of $-0.48 \pm 0.05$ $\mathrm{nm}^{-1}$.

An alternative approach to identify systems that are able to form $F d 3 m$ phases when mixed with DOPE is to use the "curvature power" $(\chi)$ of a lipid. This empirical parameter quantifies the curvature propensity of amphiphiles by calculating how the lattice parameter of the $\mathrm{H}_{\text {II }}$ phase of DOPE changes as a function of the concentration of a guest species $(j) .{ }^{31,40} \chi_{j}$ for amphiphile/lipid $(j)$ is defined as 


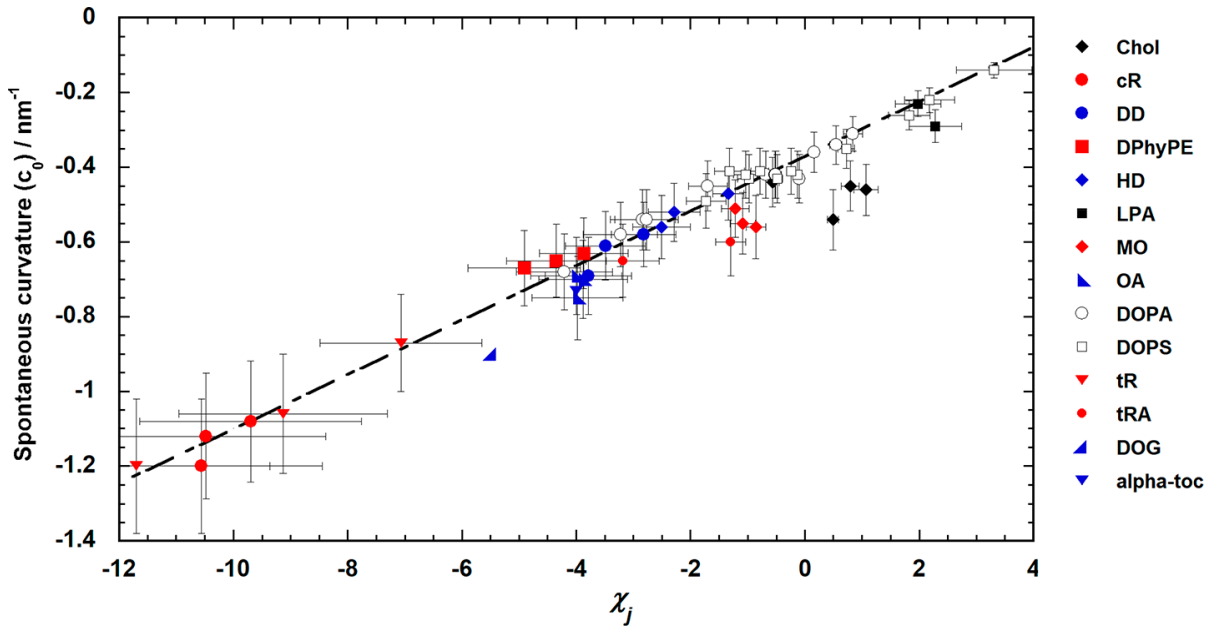

Figure 4. Universal curve of amphiphile curvature power against spontaneous curvature, showing the $\left(\chi_{j}, c_{0}\right)$ range over which $F d 3 m$ phases are observed for amphiphiles in DOPE binary mixtures (blue symbols) and systems predicted to form Fd3m phases (red symbols). The dashed line of best fit is described by eq 3 .

Table 4. Predicted Mole Fractions of Guest Lipids in DOPE at Which an Fd3m Phase Is First Expected to Appear in the Phase Diagram $^{a}$

$\begin{array}{cccccc} & \mathrm{cR} & \mathrm{tR} & \mathrm{tRA} & \mathrm{DPhyPE} & \mathrm{MO} \\ \text { mole fraction } & 0.17( \pm 0.06) & 0.19( \pm 0.06) & 0.47( \pm 0.08) & 0.44( \pm 0.08) & 0.69( \pm 0.12)\end{array}$

${ }^{a}$ Error ranges are estimated on the basis of the density of data points in our phase diagrams of DD, HD, and OA.

$$
\chi_{j}=\frac{-\left(L_{\mathrm{p}, \mathrm{PE}(37)}-L_{p, j(37)}\right)}{x_{j}}
$$

where $L_{\mathrm{p}, j(37)}$ is the lattice parameter, at $37^{\circ} \mathrm{C}$, of lipid $j$ as a guest in the $\mathrm{H}_{\mathrm{II}}$ phase of DOPE, $L_{\mathrm{p}, \mathrm{PE}(37)}$ is the lattice parameter of the $\mathrm{H}_{\text {II }}$ phase of pure DOPE, and $x_{j}$ is the mole fraction of $j$. Both lattice parameters are for phases at limiting hydration. A plot of $c_{0}$ against $\chi_{j}$ for a wide range of lipids (in DOPE mixtures) at $37{ }^{\circ} \mathrm{C}$ yields a "universal" curve, as shown in Figure 4.

This universal curve links the $c_{0}$ value of a guest lipid to its curvature power and is fitted by eq 3

$$
c_{0}=0.0731 \chi-0.3694
$$

Lipids that are known to exhibit $F d 3 m$ phases in their phase diagrams with DOPE (OA, DD, and $\mathrm{HD})$ are indicated by blue data symbols in Figure 4. These data points fall in the region of the universal curve where $\chi<-2$ and $c_{0}<-0.4$ at $37^{\circ} \mathrm{C}$. Since it is well established that DOG/DOPE mixtures form Fd3m phase it is worthwhile considering where these mixtures would lie on Figure 4. To do this we need to use a $c_{0}$ value and lattice parameter data collected at room temperature, ${ }^{44}$ since these data are not available at $37^{\circ} \mathrm{C}$. These calculations show that for a DOG/DOPE (1:9) mixture $\chi=-5.5$, where the spontaneous curvature of DOG is $-0.91 \mathrm{~nm}^{-1}$. A similar calculation can be made for data obtained at $22{ }^{\circ} \mathrm{C}$ for $\alpha$-tocopherol $\left(c_{0}=-0.73\right.$ $\left.\mathrm{nm}^{-1}\right)$ in DOPE. ${ }^{55}$ This gives $\chi=-4.0$ at (1:9) $\alpha$-tocopherol in DOPE. In Figure 4, these data points fall close to the line of best fit (eq 3), adding further evidence for our supposition that a $F d 3 m$ forming compound will occupy a range of the curve where $\chi<-2$ and $c_{0}<-0.4$ at $37{ }^{\circ} \mathrm{C}$.

Interestingly several other lipid species fall on the universal curve in the region where $\chi<-2$ and $c_{0}<-0.4$, these are $c \mathrm{R}$, tR, tRA, DPhyPE, and MO (red symbols). To the best of our knowledge $F d 3 m$ phases for these materials have yet to be discovered in DOPE however this analysis predicts that these lipid species will also form $\mathrm{Fd} 3 \mathrm{~m}$ phases in DOPE mixtures. The value of $c_{0 \text {; crit }}$ estimated above can be used as a metric for predicting the mole fraction of guest lipid in DOPE at which an Fd3m phase might first be expected to occur, as shown in Table 4. We note that MO has two stereoisomers and that in principle these molecules might have subtly different curvature powers.

If our proposed metric of $\mathrm{Fd} 3 \mathrm{~m}$ phase occurrence is correct, it is worth considering at what point it would breakdown. Figure 4 also shows that under some conditions DOPA/DOPE mixtures have $\chi<-2$ and $c_{0}<-0.4$, suggesting that in these mixtures the curvature power would favor the $F d 3 m$ phase. However, as we have previously discussed, the spontaneous curvatures of these lipids is highly dependent on the presence of divalent cations, headgroup-headgroup repulsions, and the DOPA/DOPE lipid ratio. Competition between these factors, as the composition of charged lipid/DOPE increases, means that at low composition in the DOPE matrix where the molecular shapes of some charged lipids may favor tighter curvatures initially, headgroup-headgroup repulsions will ultimately drive less tight curvature as the amount of charged lipid increases. Thus, our supposition that lipids in DOPE mixtures with $\chi<-2$ and $c_{0}<-0.4$ will form $F d 3 m$ phases is likely to be most reliable for uncharged lipids, or in the case of anionic lipids, at a $\mathrm{pH}$ where the charged groups are protonated. Hence for $\mathrm{OA}$, which has a $\mathrm{pK}$ a of $9.85,{ }^{56}$ protonated $\mathrm{OA}$ molecules are the dominant chemical form at $\mathrm{pH} 7$ in water. This explains why data for the OA/DOPE mixtures fall on the line of eq 3 in the range of $\chi$ and $c_{0}$ values where $F d 3 m$ phases form. However, we note that the $\mathrm{p} K_{\mathrm{a}}$ of amphiphiles in lipidic aggregates can vary when compared to their $\mathrm{p} K_{\mathrm{a}}$ in the bulk aqueous phase as is likely the case in OA/ DOPC $^{31}$ mixtures that form lamellar phases. 


\section{CONCLUSIONS}

Here we report for the first time the phase diagrams of DD/ DOPE and HD/DOPE and provide a detailed study of the range of stability and the structural dimension of the $\mathrm{H}_{\mathrm{II}}$ and $\mathrm{Fd} 3 \mathrm{~m}$ phases that these binary mixtures form. Comparing our data to the previously studied OA/DOPE system we have demonstrated that DD and HD broadly show similar phase behavior to that of OA when dispersed in DOPE. An analysis of the curvature power of a range of amphiphiles that form $F d 3 m$ phases leads us to predict that $\mathrm{cR}, \mathrm{tR}$ and DPhyPE will also form $\mathrm{Fd} 3 \mathrm{~m}$ phases in binary mixtures with DOPE.

Given the propensity of DD and HD to drive phospholipid assemblies to increasingly tight curvatures, it is interesting to consider if this physical property might be related to the mechanism of oxylipins in biological defense. It is clear from the work presented here that the inclusion of $\mathrm{DD}$ or $\mathrm{HD}$ in biological membranes will have dramatic effects on membrane curvature elastic stress and membrane stored elastic energy, which are both functions of the lipid spontaneous curvature. ${ }^{18}$ Given the apparent requirement for cells to maintain the biophysical parameters (membrane order ${ }^{36,57}$ and curvature elastic stress ${ }^{58,59}$ for example) of their membranes constant, in response to environmental stimuli such as chemicals, temperature, and pressure, as discussed, ${ }^{36}$ it is a possibilty that oxylipin release might disrupt this process. In this sense, the oxylipins would be analogous to the cytotoxic type I amphiphiles we have previously implicated as reducing in vivo membrane curvature elastic stress, ${ }^{60,61}$ however in the specific case of oxylipins these compounds would increase membrane curvature elastic stress.

\section{ASSOCIATED CONTENT}

\section{S Supporting Information}

The Supporting Information is available free of charge on the ACS Publications website at DOI: 10.1021/acs.langmuir.7b02998.

Sample SAXRD image and traces, plots of lattice parameters for $\mathrm{HD} / \mathrm{DOPE}$ and $\mathrm{DD} / \mathrm{DOPE}$ mixtures in $\mathrm{H}_{\mathrm{II}}$ and $\mathrm{Fd} 3 \mathrm{~m}$ geometries obtained at 37 and $52{ }^{\circ} \mathrm{C}$, respectively, tables of the lattice parameters obtained for all mixtures at all temperatures studied, background methodology on spontaneous curvature determinations (PDF)

\section{AUTHOR INFORMATION}

\section{Corresponding Authors}

*E-mail: gza@soton.ac.uk.

*E-mail: M.Dymond@brighton.ac.uk.

ORCID

Marcus K. Dymond: 0000-0002-9903-4993

Tommy Nylander: 0000-0001-9420-2217

\section{Notes}

The authors declare no competing financial interest.

\section{ACKNOWLEDGMENTS}

G.S.A. thanks B. Van Mooy for discussions about the biological roles of oxylipins. D.P. and R.J.G. gratefully acknowledge the award of studentships by Chemistry and by the University of Southampton Life Sciences Interfaces Forum, respectively. MAX-lab is acknowledged for the beam time provided under proposals 20120231, 20120338, and 20130326. T.N. acknowledges financial support from the Swedish Research Council and
M.K.D. acknowledges capital support from the School of Pharmacy and Biomolecular Sciences.

\section{REFERENCES}

(1) Mosblech, A.; Feussner, I.; Heilmann, I. Oxylipins: Structurally Diverse Metabolites from Fatty Acid Oxidation. Plant Physiol. Biochem. 2009, 47 (6), 511-517.

(2) Andreou, A.; Brodhun, F.; Feussner, I. Biosynthesis of Oxylipins in Non-Mammals. Prog. Lipid Res. 2009, 48 (3-4), 148-170.

(3) Rizzo, W. B. Fatty Aldehyde and Fatty Alcohol Metabolism: Review and Importance for Epidermal Structure and Function. Biochim. Biophys. Acta, Mol. Cell Biol. Lipids 2014, 1841 (3), 377-389.

(4) Meighen, E. A. Molecular Biology of Bacterial Bioluminescence. Microbiol. Rev. 1991, 55 (1), 123-142.

(5) Deng, W.; Hamilton-kemp, T. R.; Nielsen, M. T.; Andersen, R. A.; Collins, G. B.; Hildebrand, D. F. Effects of Six-Carbon Aldehydes and Alcohols on Bacterial Proliferation. J. Agric. Food Chem. 1993, 41, 506-510.

(6) Brodhun, F.; Feussner, I. Oxylipins in Fungi. FEBS J. 2011, 278 (7), 1047-1063.

(7) Ribalet, F.; Intertaglia, L.; Lebaron, P.; Casotti, R. Differential Effect of Three Polyunsaturated Aldehydes on Marine Bacterial Isolates. Aquat. Toxicol. 2008, 86 (2), 249-255.

(8) Ribalet, F.; Vidoudez, C.; Cassin, D.; Pohnert, G.; Ianora, A.; Miralto, A.; Casotti, R. High Plasticity in the Production of DiatomDerived Polyunsaturated Aldehydes under Nutrient Limitation: Physiological and Ecological Implications. Protist 2009, 160 (3), 444-451.

(9) Leflaive, J.; Ten-Hage, L. Chemical Interactions in Diatoms: Role of Polyunsaturated Aldehydes and Precursors. New Phytol. 2009, 184 (4), 794-805.

(10) Adolph, S.; Bach, S.; Blondel, M.; Cueff, A.; Moreau, M.; Pohnert, G.; Poulet, S. A.; Wichard, T.; Zuccaro, A. Cytotoxicity of Diatom-Derived Oxylipins in Organisms Belonging to Different Phyla. J. Exp. Biol. 2004, 207 (17), 2935-2946.

(11) Rizzo, W. B. Sjögren-Larsson Syndrome: Molecular Genetics and Biochemical Pathogenesis of Fatty Aldehyde Dehydrogenase Deficiency. Mol. Genet. Metab. 2007, 90 (1), 1-9.

(12) Marchitti, S. A.; Brocker, C.; Stagos, D.; Vasiliou, V. Non-P450 Aldehyde Oxidizing Enzymes: The Aldehyde Dehydrogenase Superfamily. Expert Opin. Drug Metab. Toxicol. 2008, 4 (6), 697-720.

(13) Tsaloglou, M.-N.; Attard, G. S.; Dymond, M. K. The Effect of Lipids on the Enzymatic Activity of 6-Phosphofructo-1-Kinase from B. Stearothermophilus. Chem. Phys. Lipids 2011, 164 (8), 713-721.

(14) Attard, G. S.; Templer, R. H.; Smith, W. S.; Hunt, A. N.; Jackowski, S. Modulation of CTP:phosphocholine Cytidylyltransferase by Membrane Curvature Elastic Stress. Proc. Natl. Acad. Sci. U. S. A. 2000, 97 (16), 9032-9036.

(15) Das, S.; Rand, R. P. Modification by Diacylglycerol of the Structure and Interaction of Various Phospholipid Bilayer Membranes. Biochemistry 1986, 25 (10), 2882-2889.

(16) Mariani, P.; Luzzati, V.; Delacroix, H. Cubic Phases of LipidContaining Systems. Structure Analysis and Biological Implications. J. Mol. Biol. 1988, 204, 165-188.

(17) Seddon, J.; Bartle, E.; Mingins, J. Inverse Cubic LiquidCrystalline Phases of Phospholipids and Related Lyotropic Systems. J. Phys.: Condens. Matter 1990, 2, SA285-SA290.

(18) Seddon, J. M. Structure of the Inverted Hexangonal (HII) Phase, and Non-Lamellar Phase-Transitions of Lipids. Biochim. Biophys. Acta, Rev. Biomembr. 1990, 1031, 1-69.

(19) Luzzati, V.; Vargas, R.; Gulik, A.; Mariani, P.; Seddon, J. M.; Rivas, E. Lipid Polymorphism: A Correction. The Structure of the Cubic Phase of Extinction Symbol Fd- Consists of Two Types of Disjointed Reverse Micelles Embedded in a Three-Dimensional Hydrocarbon Matrix. Biochemistry 1992, 31, 279-285.

(20) Takahashi, H.; Hatta, I.; Quinn, P. J. Cubic Phases in Hydrated 1:1 and 1:2 Dipalmitoylphosphatidylcholine-Dipalmitoylglycerol Mixtures. Biophys. J. 1996, 70 (3), 1407-1411. 
(21) Huang, Z.; Seddon, J. M.; Templer, R. H. An Inverse Micellar Fd3m Cubic Phase Formed by Hydrated Phosphatidylcholine/fatty Alcohol Mixtures. Chem. Phys. Lipids 1996, 82 (1), 53-61.

(22) Seddon, J. M.; Zeb, N.; Templer, R. H.; McElhaney, R. N.; Mannock, D. A. An Fd3m Lyotropic Cubic Phase in a Binary Glycolipid/Water System. Lipids 1996, 7463 (8), 5250-5253.

(23) Koynova, R.; Tenchov, B.; Rapp, G. Mixing Behavior of Saturated Short-Chain Phosphatidylcholines and Fatty Acids Eutectic Points, Liquid and Solid Phase Immiscibility, Non-Lamellar Phases. Chem. Phys. Lipids 1997, 88, 45-61.

(24) Minamikawa, H.; Hato, M. Reverse Micellar Cubic Phase in a Phytanyl-Chained Glucolipid/Water System. Langmuir 1998, 14, 4503-4509.

(25) Seddon, J. M.; Robins, J.; Gulik-krzywicki, T.; Delacroix, H. Inverse Micellar Phases of Phospholipids and Glycolipids Invited Lecture. Phys. Chem. Chem. Phys. 2000, 2, 4485-4493.

(26) Pouzot, M.; Mezzenga, R.; Leser, M.; Sagalowicz, L.; Guillot, S.; Glatter, O. Structural and Rheological Investigation of $F d 3 m$ Inverse Micellar Cubic Phases. Langmuir 2007, 23 (19), 9618-9628.

(27) Barauskas, J.; Cervin, C.; Tiberg, F.; Johnsson, M. Structure of Lyotropic Self-Assembled Lipid Nonlamellar Liquid Crystals and Their Nanoparticles in Mixtures of Phosphatidyl Choline and AlphaTocopherol (Vitamin E). Phys. Chem. Chem. Phys. 2008, 10 (43), 6483-6485.

(28) Mulet, X.; Templer, R. H.; Woscholski, R.; Ces, O. Evidence That Phosphatidylinositol Promotes Curved Membrane Interfaces. Langmuir 2008, 24 (16), 8443-8447.

(29) Bitan-Cherbakovsky, L.; Yuli-Amar, I.; Aserin, A.; Garti, N. Solubilization of Vitamin E into H(II) LLC Mesophase in the Presence and in the Absence of Vitamin C. Langmuir 2010, 26 (5), $3648-3653$.

(30) Yaghmur, A.; Sartori, B.; Rappolt, M. Self-Assembled Nanostructures of Fully Hydrated Monoelaidin-Elaidic Acid and Monoelaidin-Oleic Acid Systems. Langmuir 2012, 28 (26), 1010510119.

(31) Gillams, R. J.; Nylander, T.; Plivelic, T. S.; Dymond, M. K.; Attard, G. S. Formation of Inverse Topology Lyotropic Phases in Dioleoylphosphatidylcholine/oleic Acid and Dioleoylphosphatidylethanolamine/oleic Acid Binary Mixtures. Langmuir 2014, 30 (12), 3337-3344.

(32) Marsh, D. Elastic Curvature Constants of Lipid Monolayers and Bilayers. Chem. Phys. Lipids 2006, 144 (2), 146-159.

(33) Marsh, D. Lateral Pressure Profile, Spontaneous Curvature Frustration, and the Incorporation and Conformation of Proteins in Membranes. Biophys. J. 2007, 93 (11), 3884-3899.

(34) Helfrich, W. Elastic Properties of Lipid Bilayers: Theory and Possible Experiments. Z. Naturforsch., C: J. Biosci. 1973, 28 (11-12), 693-703.

(35) Dymond, M. K. Mammalian Phospholipid Homeostasis: Evidence That Membrane Curvature Elastic Stress Drives Homeoviscous Adaptation in Vivo. J. R. Soc., Interface 2016, 13, 20160228.

(36) Dymond, M. K. Mammalian Phospholipid Homeostasis: Homeoviscous Adaptation Deconstructed by Lipidomic Data Driven Modelling. Chem. Phys. Lipids 2015, 191, 136-146.

(37) Corsi, J.; Dymond, M. K.; Ces, O.; Muck, J.; Zink, D.; Attard, G. S. DNA That Is Dispersed in the Liquid Crystalline Phases of Phospholipids Is Actively Transcribed. Chem. Commun. (Cambridge, $U$. K.) 2008, No. 20, 2307-2309.

(38) Kozlov, M. M. Determination of Lipid Spontaneous Curvature from X-Ray Examinations of Inverted Hexagonal Phases. Methods Mol. Biol. 2007, 400, 355-366.

(39) Yaghmur, A.; Glatter, O. Characterization and Potential Applications of Nanostructured Aqueous Dispersions. Adv. Colloid Interface Sci. 2009, 147-148 (C), 333-342.

(40) Angelov, B.; Angelova, A.; Mutafchieva, R.; Lesieur, S.; Vainio, U.; Garamus, V. M.; Jensen, G. V.; Pedersen, J. S. SAXS Investigation of a Cubic to a Sponge $\left(\mathrm{L}_{3}\right)$ Phase Transition in Self-Assembled Lipid Nanocarriers. Phys. Chem. Chem. Phys. 2011, 13 (8), 3073-3081.
(41) Angelov, B.; Garamus, V. M.; Drechsler, M.; Angelova, A. Structural Analysis of Nanoparticulate Carriers for Encapsulation of Macromolecular Drugs. J. Mol. Liq. 2017, 235, 83-89.

(42) Angelov, B.; Angelova, A.; Filippov, S. K.; Drechsler, M.; Štěpánek, P.; Lesieur, S. Multicompartment Lipid Cubic Nanoparticles with High Protein Upload: Millisecond Dynamics of Formation. ACS Nano 2014, 8 (5), 5216-5226.

(43) Angelova, A.; Angelov, B.; Drechsler, M.; Garamus, V. M.; Lesieur, S. Protein Entrapment in PEGylated Lipid Nanoparticles. Int. J. Pharm. 2013, 454 (2), 625-632.

(44) Leikin, S.; Kozlov, M.; Fuller, N.; Rand, R. Measured Effects of Diacylglycerol on Structural and Elastic Properties of Phospholipid Membranes. Biophys. J. 1996, 71, 2623-2632.

(45) Kollmitzer, B.; Heftberger, P.; Rappolt, M.; Pabst, G. Monolayer Spontaneous Curvature of Raft-Forming Membrane Lipids. Soft Matter 2013, 9 (45), 10877.

(46) Dymond, M. K.; Gillams, R. J.; Parker, D. J.; Burrell, J.; Labrador, A.; Nylander, T.; Attard, G. S. Lipid Spontaneous Curvatures Estimated from Temperature-Dependent Changes in Inverse Hexagonal Phase Lattice Parameters: Effects of Metal Cations. Langmuir 2016, 32 (39), 10083-10092.

(47) Hyde, S. T. Identification of Lyotropic Liquid Crystalline Mesophases. Handbook of Applied Surface and Colloid Chemistry; Wiley, 2001; pp 299-332.

(48) Labrador, A.; Cerenius, Y.; Svensson, C.; Theodor, K.; Plivelic, T. The Yellow Mini-Hutch for SAXS Experiments at MAX IV Laboratory. J. Phys.: Conf. Ser. 2013, 425, 072019.

(49) Annibal, A.; Schubert, K.; Wagner, U.; Hoffmann, R.; Schiller, J.; Fedorova, M. New Covalent Modifications of Phosphatidylethanolamine by Alkanals: Mass Spectrometry Based Structural Characterization and Biological Effects. J. Mass Spectrom. 2014, 49 (7), 557569.

(50) Plack, P. A.; Pritchard, D. J. Schiff Bases Formed from Retinal and Phosphatidylethanolamine, Phosphatidylserine, Ethanolamine or Serine. Biochem. J. 1969, 115 (5), 927-934.

(51) Stadelmann-Ingrand, S.; Pontcharraud, R; Fauconneau, B. Evidence for the Reactivity of Fatty Aldehydes Released from Oxidized Plasmalogens with Phosphatidylethanolamine to Form Schiff Base Adducts in Rat Brain Homogenates. Chem. Phys. Lipids 2004, 131 (1), 93-105.

(52) Groendijk, G. W. T.; Jacobs, C. W. M.; Bonting, S. L.; Damen, F. J. M. Dark Isomerization of Retinals in the Presence of Phosphatidylethanolamine. Eur. J. Biochem. 1980, 106 (1), 119-128.

(53) Guo, L.; Davies, S. S. Bioactive Aldehyde-Modified Phosphatidylethanolamines. Biochimie 2013, 95 (1), 74-78.

(54) Guo, L.; Chen, Z.; Amarnath, V.; Davies, S. S. Identification of Novel Bioactive Aldehyde-Modified Phosphatidylethanolamines Formed by Lipid Peroxidation. Free Radical Biol. Med. 2012, 53 (6), $1226-1238$.

(55) Bradford, A.; Atkinson, J.; Fuller, N.; Rand, R. P. The Effect of Vitamin E on the Structure of Membrane Lipid Assemblies. J. Lipid Res. 2003, 44 (10), 1940-1945.

(56) Kanicky, J. R.; Shah, D. O. Effect of Degree, Type, and Position of Unsaturation on the pKa of Long-Chain Fatty Acids. J. Colloid Interface Sci. 2002, 256 (1), 201-207.

(57) Sinensky, M. Homeoviscous Adaptation-a Homeostatic Process That Regulates the Viscosity of Membrane Lipids in Escherichia Coli. Proc. Natl. Acad. Sci. U. S. A. 1974, 71 (2), 522-525.

(58) Gruner, S. M. Intrinsic Curvature Hypothesis for Biomembrane Lipid Composition: A Role for Nonbilayer Lipids. Proc. Natl. Acad. Sci. U. S. A. 1985, 82 (11), 3665-3669.

(59) Dymond, M. K.; Hague, C. V.; Postle, A. D.; Attard, G. S. An in Vivo Ratio Control Mechanism for Phospholipid Homeostasis: Evidence from Lipidomic Studies. J. R. Soc., Interface 2013, 10 (80), 20120854.

(60) Dymond, M. K.; Attard, G. S.; Postle, A. D. Testing the Hypothesis That Amphiphilic Antineoplastic Lipid Analogues Act through Reduction of Membrane Curvature Elastic Stress. J. R. Soc., Interface 2008, 5 (28), 1371-1386. 
(61) Dymond, M. K.; Attard, G. S. Cationic Type I Amphiphiles as Modulators of Membrane Curvature Elastic Stress in Vivo. Langmuir 2008, 24 (20), 11743-11751. 